\title{
La Tuba Uterina: Desde Herófilo a Horacio Croxatto
}

\author{
The Uterine Tube: From Herophilus to Horacio Croxatto
}

Godoy-Guzmán, C. ${ }^{1,2}$; Fuentes, J. L. ${ }^{3}$; Osses, M. ${ }^{1}$; Toledo-Ordoñez, I. ${ }^{1}$ \& Orihuela, P.,5

GODOY-GUZMÁN, C.; FUENTES, J. L.; OSSES, M.; TOLEDO-ORDOÑEZ, I. \& ORIHUELA, P. La tuba uterina: desde Herófilo a Horacio Croxatto. Int. J. Morphol., 36(2):387-390, 2018.

RESUMEN: Las tubas uterinas (TU) son órganos tubulares fundamentales en la reproducción humana. No obstante, recién a mediados del siglo XVII con las investigaciones de Reinier De Graaf se comienza a develar su verdadera función en la reproducción. En este trabajo se resumen las principales contribuciones de Horacio Croxatto Avoni al conocimiento de la morfología y fisiología de la TU humana. Sus principales aportes tienen relación con la fisiología del transporte del cigoto y los gametos a lo largo de la TU.

PALABRAS CLAVE: Tuba uterina; Humano; Croxatto.

\section{INTRODUCCIÓN}

Las tubas uterinas (TU) son dos conductos que se extienden a lo largo del margen superior de los ligamentos anchos del útero, desde los cuernos del útero hasta la superficie del ovario (Rouvière \& Delmas, 2005). Este órgano posee una gran importancia reproductiva, cumpliendo funciones tales como: recoger al ovocito, controlar el ascenso de los espermatozoides, es el sitio donde ocurre generalmente la fecundación, transporta y nutre al cigoto en las primeras etapas de su desarrollo, entre otras (Croxatto, 2002). Se han descrito cuatro segmentos anatómicos: infundíbulo, ampolla, istmo y porción intramural. Por otro lado, su pared está compuesta por 3 capas: mucosa, muscular y serosa. La mucosa está recubierta por un epitelio columnar simple con células ciliadas y células no ciliadas (peg), las cuales descansan sobre una lámina basal y una lámina propia subyacente. La capa muscular corresponde a células musculares lisas donde se puede distinguir una capa circular interna y otra longitudinal externa. La serosa está revestida por un epitelio simple plano, con tejido conectivo subyacente donde se pueden observar grandes vasos sanguíneos y nervios.

El entendimiento de la función de la TU ha sido paralelo a los avances en el conocimiento sobre el desarrollo embrionario. Los primeros hallazgos datan de la antigua Grecia y fueron posibles a partir de la observación directa de material anatómico no tratado y posteriormente debido al desarrollo de aparatos de magnificación más sofisticados, como los microscopios. Sin embargo, no es hasta mediados del siglo XVII con los trabajos de Reinier De Graaf que se comienza a develar su verdadera función e importancia en el proceso reproductivo. Cabe hacer notar que desde mediados del siglo XX los hallazgos morfológicos obtenidos a través del uso de la microscopía electrónica de transmisión (MET), estimularon toda una serie de investigaciones sobre la fisiología de la TU. En este trabajo se resumen las principales contribuciones de Horacio Croxatto Avoni al conocimiento de la morfología y fisiología de la TU humana.

\section{ALGUNOS ANTECEDENTES HISTÓRICOS}

El actual conocimiento sobre la anatomía y fisiología de la TU ha sido posible gracias al trabajo de múltiples científicos tales como Aristóteles (384-322 A.C.), quién propone que el embrión se origina a partir de la mezcla entre el semen masculino y el semen femenino (flujo uterino o menstrual), sin embargo, no pone atención al rol de la TU. Esta hipótesis coincide con una de las primeras descripciones de la TU humana hecha por Herofilo (335-280 A.C.), médico griego, quien en su tratado sobre partería señala la presencia de 2 conductos que posiblemente transportan el semen femenino desde los ovarios a la vejiga (Wells, 1948; Kothary \& Kothary, 1975; Hunter, 1988). Posteriormente, Galeno (130-200 D.C.) describió que los conductos pares señalados por Herophilus se conectan con el útero y no con la vejiga.

${ }^{1}$ Unidad de Histología (CIBAP), Escuela de Medicina, Universidad de Santiago de Chile, (USACH), Santiago, Chile.

${ }^{2}$ Centro de Investigaciones Biomédicas y Aplicadas, Escuela de Medicina, Universidad de Santiago de Chile, (USACH), Santiago, Chile.

${ }^{3}$ Facultad de Medicina, Universidad Diego Portales, Santiago, Chile.

${ }^{4}$ Laboratorio de Inmunología de la Reproducción, Facultad de Química y Biología, Universidad de Santiago de Chile, (USACH), Santiago, Chile.

${ }^{5}$ Centro para el Desarrollo en Nanociencia y Nanotecnología-CEDENNA, Universidad de Santiago de Chile, (USACH), Santiago, Chile. 
En 1561 Gabriel Fallopio (1523-1562) publica en Venecia su libro Observationes Anatomicae. Su aporte consiste en una descripción detallada de la TU, sus diferentes porciones, con uno de sus extremos distales abiertos hacia el abdomen y su conexión con el útero (Tripathy, 2013).

Uno de los principales científicos que contribuyeron al conocimiento de la TU, fue Reiner De Graaf (1641-1673) quién es considerado el fundador de la biología reproductiva moderna (Jocelyn \& Setchell, 1972). Destaca el trabajo titulado "De mulierum organis generationi inservientibus" publicado en 1672, donde señala (Houtzager, 2000): la presencia de folículos en el ovario, que el "aura seminalis" penetra el ovario y al folículo ocurriendo la fecundación, y el posterior transporte del huevo desde el ovario al útero a través de la TU. De Graaf fue el primero en identificar la verdadera función de la TU, y cuestiona la doctrina aristotélica de que el embrión se origina en el útero como resultado de activación del flujo menstrual por el semen masculino (Jay, 2000). Posteriormente, la invención del microscopio permitió confirmar algunas de las hipótesis planteadas por De Graaf en el campo reproductivo. En esta línea, destaca el descubrimiento del espermatozoide por Antoine Van Leeuwenhoek (1632-1723) en 1677 y del ovocito por Karl Ernst Von Baer (1792-1876) en 1827.

La invención del MET entre 1931 y 1933 por Ernst Ruska abrió nuevos horizontes de investigación en las ciencias morfológicas, no siendo la excepción la TU (Patek, 1974; Rumery \& Eddy, 1974; Stalheim et al., 1975). Los hallazgos obtenidos a partir de la MET estimularon el desarrollo de diversas líneas de investigación sobre la fisiología de la TU tales como; la contractilidad in vivo, estudios farmacológicos, transporte de gametos, composición del fluido, entre otros (Hunter).

Contribuciones de Horacio Croxatto. Horacio Croxatto Avoni (Chile, 14 de Julio de 1936) médico, biólogo y fisiólogo experto en reproducción humana y métodos anticonceptivos, es uno de los principales investigadores del mundo sobre la estructura y fisiología de la TU humana, su trabajo está avalado por alrededor de 234 publicaciones, 61 capítulos de libros y 17 patentes (Fig. 1).

En 1961 obtiene el título de Médico en la Pontificia Universidad Católica de Chile (PUC), realizó estudios posdoctorales con el profesor Charles H. Sawyer en el National Institute of Health de la Universidad de California en Los Ángeles entre 1964 a 1966. En 1965 fue becado por Population Council de la Universidad de Rockefeller de Nueva York, una fundación sin fines de lucro que tenía por misión el enfrentar científicamente el problema de la explosión demográfica. El Dr. Sheldon Segal le encargó la tarea

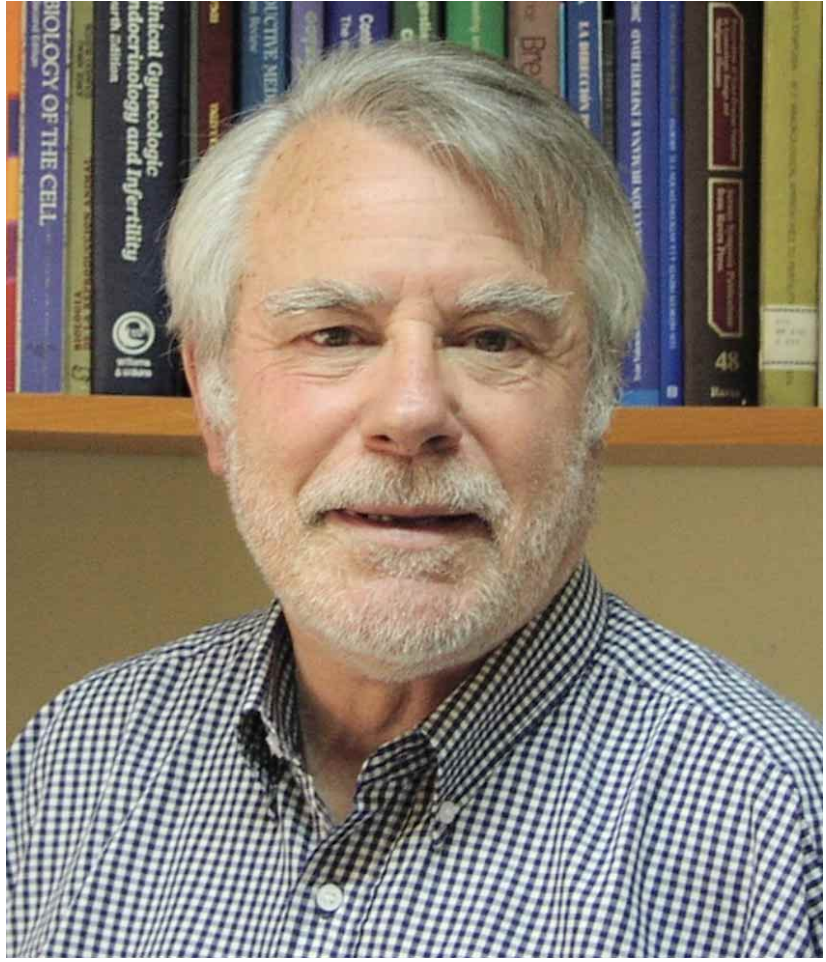

Fig. 1. Horacio Croxatto Avoni (Chile, 14 de Julio 1936) médico, biólogo y fisiólogo experto en reproducción humana y métodos anticonceptivos, es uno de los principales investigadores del mundo sobre la estructura y fisiología de la TU humana. (https:// www.academiasexologia.org/croxatto.html, consultada 12-01-2018).

de desarrollar un método hormonal inyectable de larga duración que pudiera reemplazar la ingesta diaria de una píldora. A partir de sus investigaciones en modelos animales crea el implante subdérmico, método anticonceptivo que previene el embarazo y ha beneficiado a millones de mujeres en todo el mundo. A continuación, se señalan algunas de las labores que ha desempeñado:

- Miembro del Comité Internacional para la investigación en anticoncepción del Population Council, Nueva York (1973- a la actualidad).

- Presidente del Instituto Chileno de Medicina Reproductiva (ICMER) entre 1985 a 2008.

- Profesor Titular. PUC (1968-2005).

- Profesor de la Facultad de Química y Biología de la Universidad de Santiago de Chile (2005-2011).

- Profesor honorario de la Universidad de Chile y Universidad de Valparaíso.

- Actualmente, dirige el Center for Integrative Medicine and Innovative Science (CIMIS) de la Universidad Andrés Bello (2012- a la actualidad).

Principales Hallazgos sobre la TU humana. Horacio Croxatto ha realizado numerosos aportes al conocimiento 
de la morfología y fisiología del transporte del cigoto y gametos a través de la TU humana y de algunos modelos animales (Croxatto \& Villalón, 1995; Croxatto, 2002; Croxatto, 1997; Pérez Sánchez et al., 2011).

Determinó que el periodo de transporte a lo largo de la TU se inicia inmediatamente después de la ovulación con la incorporación del ovocito al lumen de la tuba uterina. El comienzo del transporte depende de la localización regional del ovocito, estableciéndose un rango entre minutos a horas (Croxatto et al., 1989). En esta línea, sugiere que, en la mujer, el folículo vacía su contenido a la cavidad peritoneal, y el ovocito es recuperado por la TU gracias a la contracción del ligamento tubo-ovárico deslizando la fimbria sobre la superficie del ovario. Eventualmente, si este mecanismo falla, es posible que la fimbria capte el cúmulo oophorus directamente desde el fondo de saco recto-uterino, ya que ha demostrado que ésta recoge fácilmente microesferas inyectadas en la cavidad peritoneal a través de la porción posterior del fondo vaginal (Diaz et al., 1976).

Además, determinó en mujeres la duración del transporte del ovocito a lo largo de la TU. Fue estimado en estudios realizados en mujeres esterilizadas de las cuales se recuperaron ovocitos desde la TU y la cavidad uterina; en todos los casos se conocía el tiempo transcurrido desde el pico de LH hasta el momento de la intervención. Señala que considerando que a las 96 horas la mitad de los ovocitos se recuperaron del útero, que la ovulación puede ocurrir 17 horas después del pico de LH y asumiendo que la captación del cúmulo oophorus se completa en la primera hora después de la ovulación, se estimó que el tránsito del ovocito por la TU dura aproximadamente 80 horas. Además, señala que la mayor parte del tiempo el ovocito permanece en la ampolla (90\% del tiempo) y que atraviesa el istmo en menos de 10 horas (Croxatto et al., 1978; Croxatto et al., 1989; Croxatto et al., 1996). Además, describe que el cigoto viaja a través de la TU hasta llegar a la cavidad uterina 4 días después de la ovulación en el estadio de mórula (Croxatto et al., 1972).

El transporte del ovocito en la ampolla depende principalmente de la acción de las células ciliadas que baten hacia el útero, mientras que el transporte a través del istmo depende principalmente de la contracción de la capa muscular (Croxatto, 2002). En el istmo, el ovocito se mueve rodeado de líquido que es transportado en una u otra dirección por cambios en los gradientes de presión intraluminal causados por ciclos de contracción y relajación de la capa muscular que ocurren simultánea y sucesivamente en distintos puntos (Moore \& Croxatto, 1988). La frecuencia del batido de los cilios cambia en el transcurso del ciclo ovárico. Se han descrito diversas sustancias que pueden modificar la frecuencia del batido ciliar, las cuales son sintetizadas por las células de la TU, espermáticas o embrionarias, tales como: Prostaglandinas, Factor activador de plaquetas (PAF), ATP, $\mathrm{E}_{2}, \mathrm{E}_{1}$, entre otras (Croxatto, 2002).

También realizó experimentos para estudiar la regulación del transporte de los gametos y el cigoto a través de la TU. En este sentido, describe que la administración de altas dosis de estradiol y progesterona en una mujer inmediatamente después de la ovulación no altera la captación de ovocitos dentro del período que normalmente deben estar en el útero, sugiriendo que no tendrían un rol importante en la regulación (Croxatto, 1996). En esta línea, describió en hámster que el factor PAF de origen embrionario desencadena el paso del embrión desde de la TU al útero (Velásquez et al., 1995). Posteriormente, reforzó estos hallazgos en un estudio donde describió la expresión de PAF y su receptor (PAFr) en el epitelio y las células del estroma en la TU humana. Croxatto, además sugiere que estas células producen en respuesta a PAF de origen embrionario algún agente que actúa sobre células ciliadas o células musculares lisas o secretoras (Velásquez et al., 2001).

Destaca una de las primeras descripciones de la ultraestructura del embrión humano compuesto por 7 blastómeros y las células del cúmulo oóphorus (Pereda \& Croxatto, 1978; Motta et al., 1995). Así como también el estudio por MET de embriones humanos de 2, 4, 7 y 16 células y por microscopia de luz la morfología del ovocito secundario entre las 24 y 144 horas después del pico de LH (Ortiz et al., 1982; Pereda \& Croxatto, 1992).

También estudió en ratas la migración de los espermatozoides en la TU en distintas etapas del ciclo estral variando de acuerdo a la etapa del ciclo y dependiendo de $\mathrm{E}_{2}$ y $\mathrm{P}_{4}$. La adhesión de los espermatozoides al epitelio de la TU depende de la etapa del ciclo y segmento específico y requiere la combinación de ambas hormonas (Orihuela et al., 1999).

\section{PREMIOS}

El Dr. Croxatto ha recibido múltiples premios, destaca la cátedra presidencial en ciencias otorgada por el Gobierno de Chile en 1999 por su productividad científica. El año 2014 fue el primer latinoamericano en recibir el premio internacional de la Society of Family Planning por sus importantes logros y contribuciones en el campo de la planificación familiar. Recientemente, el año 2016 se le concedió el Premio Nacional de Ciencias Aplicadas y Tecnológicas de Chile.

AGRADECIMIENTOS. Universidad de Santiago de Chile, USACH. Agradecimientos Proyecto DICYT, Código 021501GG, Vicerrectoría de Investigación, Desarrollo e Innovación y Proyecto Basal FB0807. 
GODOY-GUZMÁN, C.; FUENTES, J. L.; OSSES, M.; TOLEDO-ORDOÑEZ, I. \& ORIHUELA, P. The Uterine Tube: From Herophilus to Horacio Croxatto. Int. J. Morphol., 36(2):387$390,2018$.

SUMMARY: The uterine tubes (UT) are fundamental tubular organs in human reproduction. However, it was not until the middle of the 17th century that Reinier De Graaf's research began to reveal its true role in reproduction. In this work the main contributions of Horacio Croxatto Avoni toward the knowledge of the morphology and physiology of the human UT are summarized. Its main contributions are related to the physiology of zygote transport and gametes throughout the UT.

\section{KEY WORDS: Uterine tube; Humane; Croxatto.}

\section{REFERENCIAS BIBLIOGRÁFICAS}

Croxatto, H. B. \& Ortiz, M. E. Oocyte Pickup and Oviductal Transport. En: Capitanio, G. L.; Asch, R. H.; De Cecco, L. \& Croce, S. (Eds.). GIFT: From Basics to Clinic. New York, Raven Press, 1989. pp.137-47.

Croxatto, H. B. \& Villalon, M. Oocyte Transport. En: Grudzinskas, J. G. \& Yovich, J. L. (Eds.). Cambridge Reviews in Human Reproduction. GametesThe Oocyte. Cambridge, Cambridge University Press, 1995. pp.253-76.

Croxatto, H. B. Gamete Transport. En: Adashi, E. Y.; Rock, J. A. \& Rosenwaks, Z. (Eds.). Reproductive Endocrinology, Surgery and Technology. Philadelphia, Lippincott-Raven Publishers, 1999. pp.385-402.

Croxatto, H. B. Physiology of gamete and embryo transport through the fallopian tube. Reprod. Biomed. Online, 4(2):160-9, 2002.

Croxatto, H. B.; Díaz, S.; Fuentealba, B.; Croxatto H. D.; Carrillo, D. \& Fabres, C. Studies on the duration of egg transport in the human oviduct. I. The time interval between ovulation and egg recovery from the uterus in normal women. Fertil. Steril., 23:447-58, 1972.

Croxatto, H. B.; Ortiz, M. E.; Díaz, S.; Hess, R.; Balmaceda, J. \& Croxatto, H. D. Studies on the duration of egg transport by the human oviduct. II. Ovum location at various intervals following luteinizing hormone peak. Am. J. Obstet. Gynecol., 132(6):629-34, 1978.

Croxatto, H. B.; Ortiz, M. E.; Forcelledo, M. L. \& Fuentealba, B. Transporte del huevo por el tracto genital y su regulación. Arch. Biol. Med. Exp., 15:309$14,1982$.

Croxatto, H. B.; Ortiz, M. E.; Villalón, M.; Cárdenas, H.; Imarai, M.; Hermoso, M.; Velásquez, L. A. \& Orihuela, P. Basic Aspects of Oviduct Function. En: Coutifaris, C. \& Mastroianni, L. New Horizons in Reproductive Medicine. Casterton, Parthenon Publishing Co., 1997. pp.233-9.

Diaz, J.; Vasquez, J.; Diaz, S.; Diaz, F. \& Croxatto, H. B. Transport of Ovum Surrogates by the Human Oviduct. En: Harper, M. J. K.; Pauerstein, C. J.; Adams, C. E.; Coutinho, E. M.; Croxatto, H. B. \& Paton, D. M. (Eds.). Ovum Transport and Fertility Regulation. Copenhagen, Scriptor, 1976. pp.404-15.

Houtzager, H. L. Reinier De Graaf and his contribution to reproductive biology. Eur. J. Obstet. Gynecol. Reprod. Biol., 90(2):125-7, 2000.

Hunter, H. F. The Fallopian Tubes: Their Role in Fertility and Infertility. Berlin, Springer-Verlag, 1988.

Jay, V. The Legacy of Reinier De Graaf. Arch. Pathol. Lab. Med, 124(8):1115-6, 2000.

Jocelyn, H. D. \& Setchell, B. P. Regnier de Graaf on the human reproductive organs. An annotated translation of Tractatus de Virorum Organis Generationi Inservientibus (1668) and De Mulierub Organis Generationi Inservientibus Tractatus Novus (1962). J. Reprod. Fertil. Suppl., 17:1-222, 1972.

Kothary, P. C. \& Kothary, S. P. Gabriele Fallopio. Int. Surg., 60(2):80-1, 1975.

Moore, G. D. \& Croxatto, H. B. Effects of delayed transfer and treatment with oestrogen on the transport of microspheres by the rat oviduct. J. Reprod. Fertil., 83(2):795-802, 1988.
Motta, P. M.; Nottola, S. A.; Pereda, J.; Croxatto, H. B. \& Familiari, G. Ultrastructure of human cumulus oophorus: a transmission electron microscopic study on oviductal oocytes and fertilized eggs. Hum. Reprod., 10(9):2361-7, 1995.

Orihuela, P. A.; Ortiz, M. E. \& Croxatto, H. B. Sperm migration into and through the oviduct following artificial insemination at different stages of the estrous cycle in the rat. Biol. Reprod., 60(4):908-13, 1999.

Ortiz, M. E.; Salvatierra, A. M.; López, J.; Fernández, E. \& Croxatto, H. B. Postovulatory aging of human ova: I. Light microscopic observations. Mol. Reprod. Dev., 6(1):11-7, 1982.

Patek, E. The epithelium of the human fallopian tube. Acta Obstet. Gynecol. Scand., 53(S31):4-28, 1974.

Pereda, J. \& Croxatto, H. B. Human preimplantation development in vivo: ultrastructural observations. Ann. Acad. Med. Singapore, 21(4):480-8, 1992.

Pereda, J. \& Croxatto, H. B. Ultrastructure of a seven-cell human embryo. Biol. Reprod., 18(3):481-9, 1978.

Pérez Sánchez, A. Obstetricia. 4ª ed. Santiago de Chile, Mediterráneo, 2011. pp.89-107.

Rouvière, H. \& Delmas, A. Anatomia Humana: Descriptiva, Topográfica y Funcional. Barcelona, Masson, 2005.

Rumery, R. E. \& Eddy, E. M. Scanning electron microscopy of the fimbriae and ampullae of rabbit oviducts. Anat. Rec., 178(1):83-101, 1974.

Stalheim, O. H.; Gallagher, J. E. \& Deyoe, B. L. Scanning electron microscopy of the bovine, equine, porcine, and caprine uterine tube (oviduct). Am. J. Vet. Res., 36(08):1069-75, 1975.

Tripathy, S. N. The Fallopian Tubes. Odisha, Jaypee Brothers Medical Publishers, 2013.

Velásquez, L. A.; Aguilera, J. G. \& Croxatto, H. B. Possible role of plateletactivating factor in embryonic signaling during oviductal transport in the hamster. Biol. Reprod., 52(6):1302-6, 1995.

Velásquez, L. A.; Maisey, K.; Fernandez, R.; Valdes, D.; Cardenas, H.; Imarai, M.; Delgado, J.; Aguilera, J. \& Croxatto, H. B. PAF receptor and PAF acetylhydrolase expression in the endosalpinx of the human Fallopian tube: possible role of embryo-derived PAF in the control of embryo transport to the uterus. Hum. Reprod., 16(8):1583-7, 2001.

Wells, W. A. Gabriel Fallopio, one of the 16th century founders of modern anatomy; also distinguished physician and surgeon, 1523-1562. Laryngoscope, 58(1):33-42, 1948.

Dirección para correspondencia:

Prof. Carlos Godoy-Guzmán

Facultad de Ciencias Médicas

Universidad de Santiago de Chile

Avda. Bdo. O'Higgins 3363

Correo 442

Santiago

CHILE

E-mail: carlos.godoy@usach.cl

Recibido : 12-01-2018

Aceptado: 30-03-2018 